\title{
Using State and National Surveillance Systems during World Equestrian Games in NC
}

\author{
Lana Deyneka ${ }^{1}$, Zachary Faigen ${ }^{1}$, Anne Hakenewerth ${ }^{1}$, Nicole Lee ${ }^{1}$, Amy Ising ${ }^{2}$, Meichun Li² \\ ${ }^{1}$ Epidemiology, NC DPH, Raleigh, North Carolina, United States, ${ }^{2}$ UNC, Chapel Hill, North Carolina, United States \\ Objective
}

To describe surveillance activities and use of existing state (NC DETECT) and national (NSSP) syndromic surveillance systems during the International Federation for Equestrian Sports (FEI) World Equestrian Games (WEG), in Mill Spring, NC from September 11 to September 23, 2018

\section{Introduction}

North Carolina hosted the 2018 FEI WEG in Polk County at the Tryon Equestrian Center in September 2018. Polk County, located in the Mountain region of Western North Carolina, is home to 20,357 people, and the population is widely distributed. Event organizers expected approximately 300,000 to 500,000 people to visit the area, with 800 horses from 71 countries taking part in the games. Providing adequate public health epidemiologic investigations and response for the large scale event in the predominantly rural area presented a challenge. The NC Surveillance \& Response Team was created to facilitate enhanced surveillance for significant public health events during the WEG, assist local public health agencies with epidemiologic investigations and response, develop public health risk assessments, and implement control measures. Surveillance data were collected from the North Carolina Electronic Disease Surveillance System (NC EDSS), North Carolina's and CDC's National syndromic surveillance systems (NC DETECT and NSSP ESSENCE), Public Health Epidemiologists from Atrium Health and Mission Hospital, and reports from the on-site medical facility (MED-1) at the Tryon Equestrian Center. The data were reviewed and summarized in internal and external situation reports.

\section{Methods}

NC DETECT collects statewide data from hospital emergency department (ED) visits and Carolinas Poison Center (CPC) calls. NC DETECT also collects data from select Urgent Care Centers (UCC) in the Charlotte area. CPC data are updated hourly, while ED data are updated twice a day. NC DETECT data were monitored daily for census (total ED visits), communicable disease syndromes, injury syndromes, and other occurrences of public health significance related to the event. The geographic areas monitored were Polk County (the location of the main event), the counties where the guests were lodging in the Western NC Region (Henderson, Transylvania, Buncombe, Rutherford, McDowell, and Cleveland), the Charlotte Metropolitan area, and statewide. Because of the large number of people from other states and countries who attended, ED surveillance was mainly conducted by hospitals so that visits were captured for all patients and not just NC residents. WEG dashboards containing ED data were created prior to the event using NC DETECT and NSSP ESSENCE systems, and were accessible to epidemiologists at the state level. NSSP syndrome queries were shared with the neighboring state (SC) public health agency. Surveillance began two weeks prior to the event to establish baseline levels for all ED visits for hospitals in Polk County and the Western NC Region. Surveillance occurred daily before the event, during the event, and for two weeks following the event to account for incubation periods of potential diseases.

\section{Results}

The 2018 Equestrian games in Western NC were affected by heavy rain and heat. The weather led to low attendance and cancellation of a few competitions. During the observation period, ED admissions and most of the mass gathering related syndromes in both NC DETECT and NSSP systems were at baseline. ED admissions for motor vehicle collisions and dehydration syndromes were above baseline for 09/19 and 09/21/18 (Figures 3-4). CPC calls and UC admissions for selected UC centers in the Charlotte area were also monitored, and were at baseline.

\section{Conclusions}

NC DETECT and NSSP Dashboards provided effective and timely surveillance for the WEG event to assist local public health in the rural NC area with epidemiologic investigations and appropriate response. NC DETECT's CPC and UC data provided additional valuable information, and complemented ED surveillance during the mass gathering event. Syndromic surveillance 
ISDS 2019 Conference Abstracts

became essential during WEG, as NC DPH deployment plans and resource availability changed when Hurricane Florence bore down on the region.

\section{Acknowledgement}

Anna Waller, ScD, NC DETECT Principal Investigator Clifton Barnett, MSIS, NC DETECT Data Quality Manager Dennis Falls, NC DETECT ETL Manager / Information Security Officer Bill Jones, NC DPH, Public Health Program Manager

\section{References}

1. Joseph S. Lombardo, Carol A. Sniegoski, Wayne A. Loschen, Matthew Westercamp, Michael Wade, Shandy Dearth, and Guoyan Zhang Public Health Surveillance for Mass Gatherings Johns Hopkins APL Technical Digest, Volume 27, Number 4 (2008)

2. Kaiser R, Coulombier D. 2006. Epidemic intelligence during mass gatherings. Euro Surveill. 11(12):E061221.3. PubMed

3. Ising A, Li M, Deyneka L, Vaughan-Batten H, Waller A. Improving syndromic surveillance for nonpower users: NC DETECT dashboards. Emerging Health Threats Journal 2011, 4: 11702 - DOI: 10.3402/ehtj.v4i0.11702

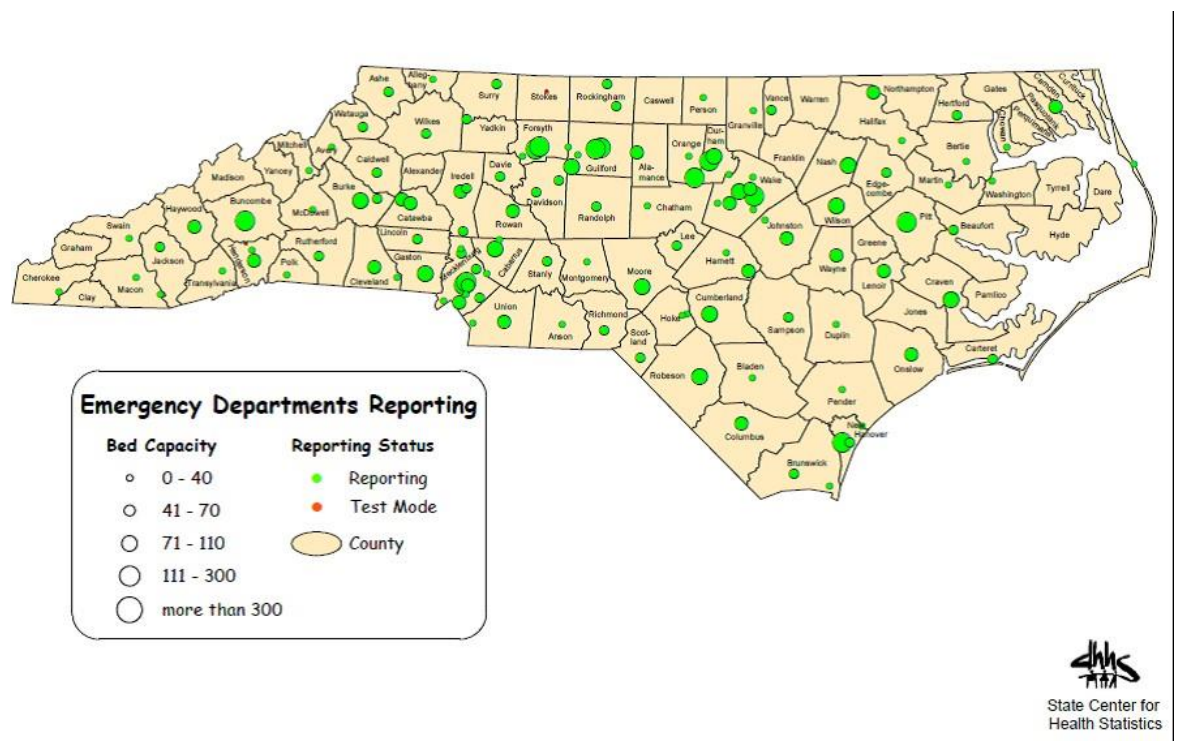

Figure 1 


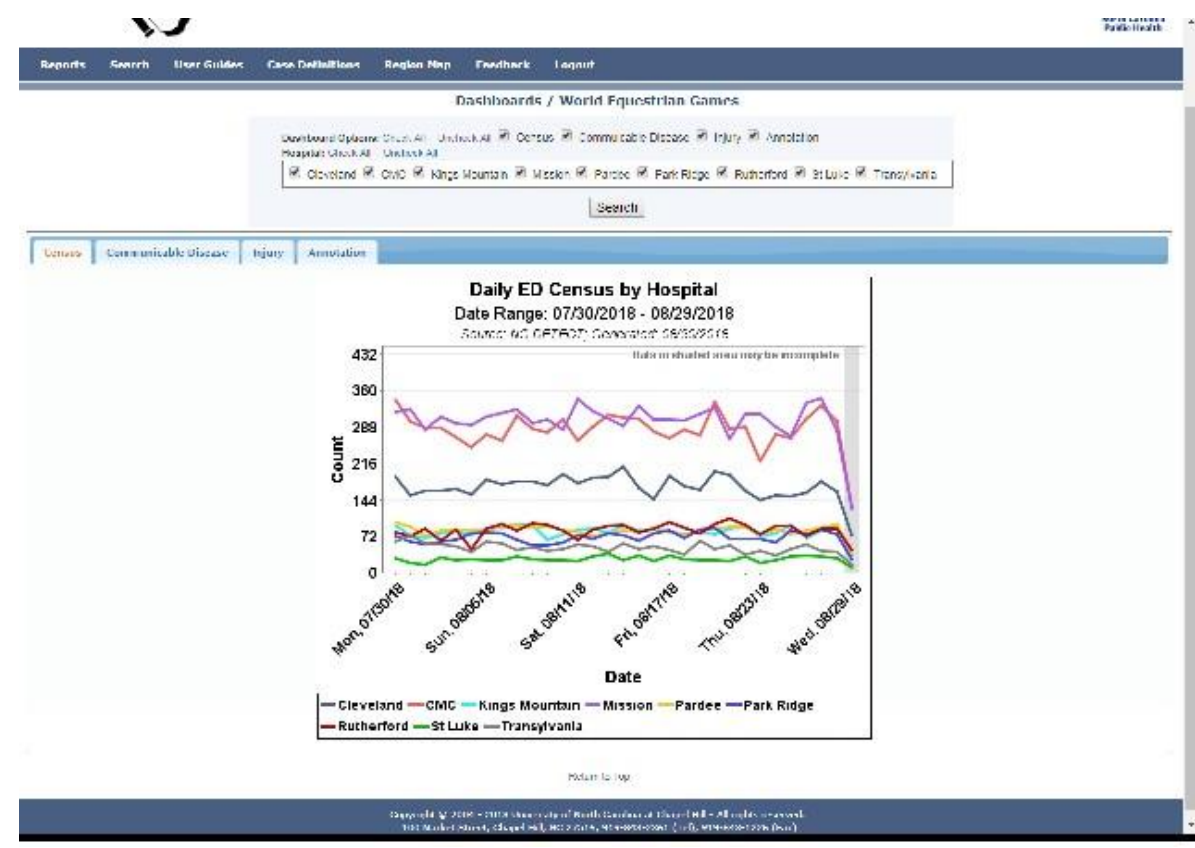

Figure 2

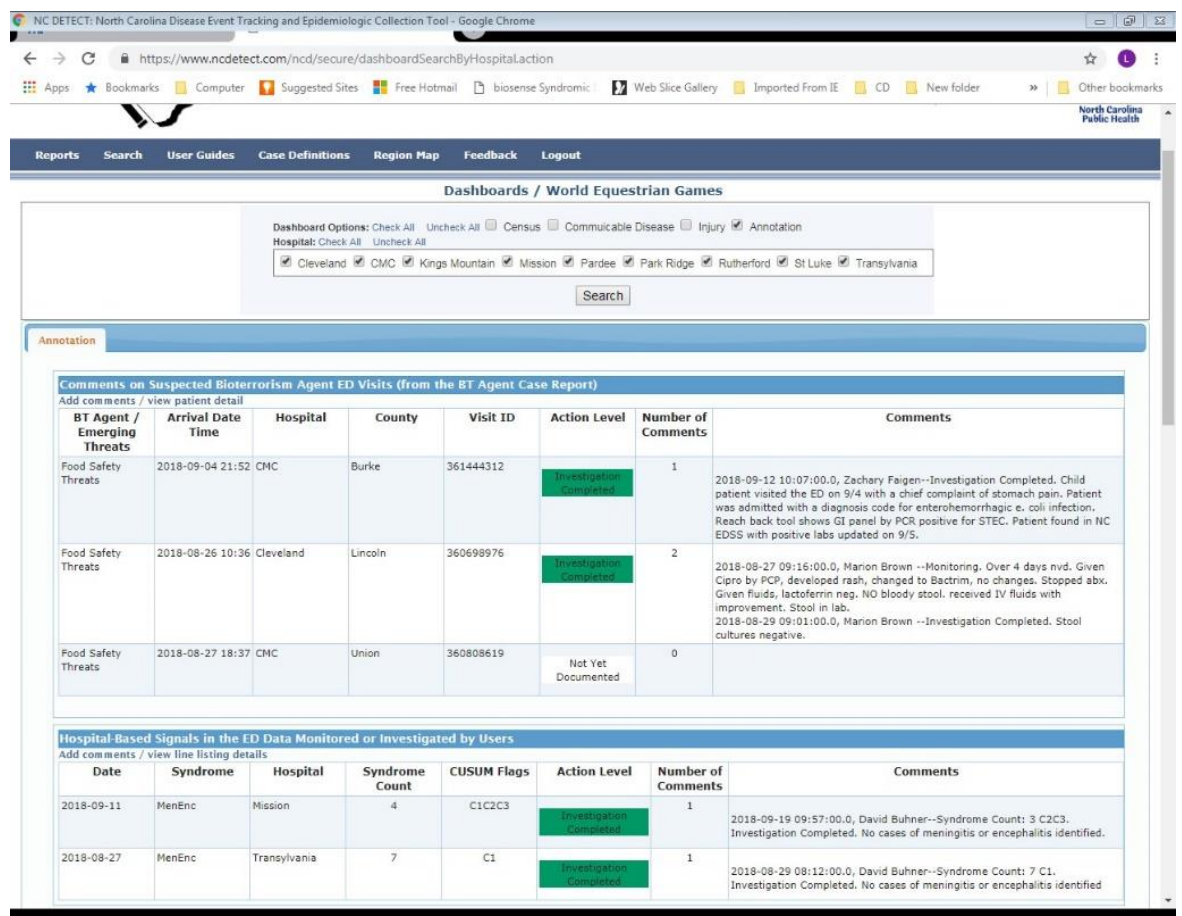

Figure 3 

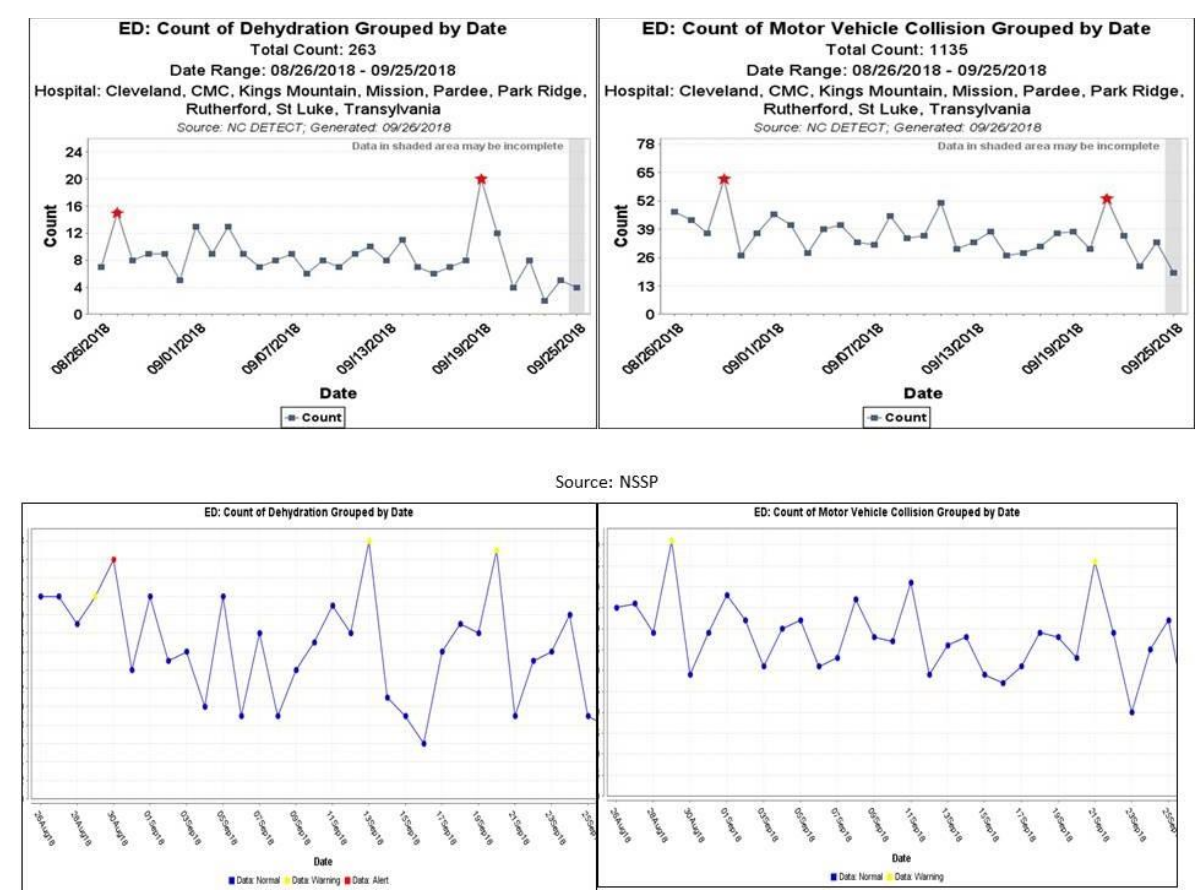

Figure 4

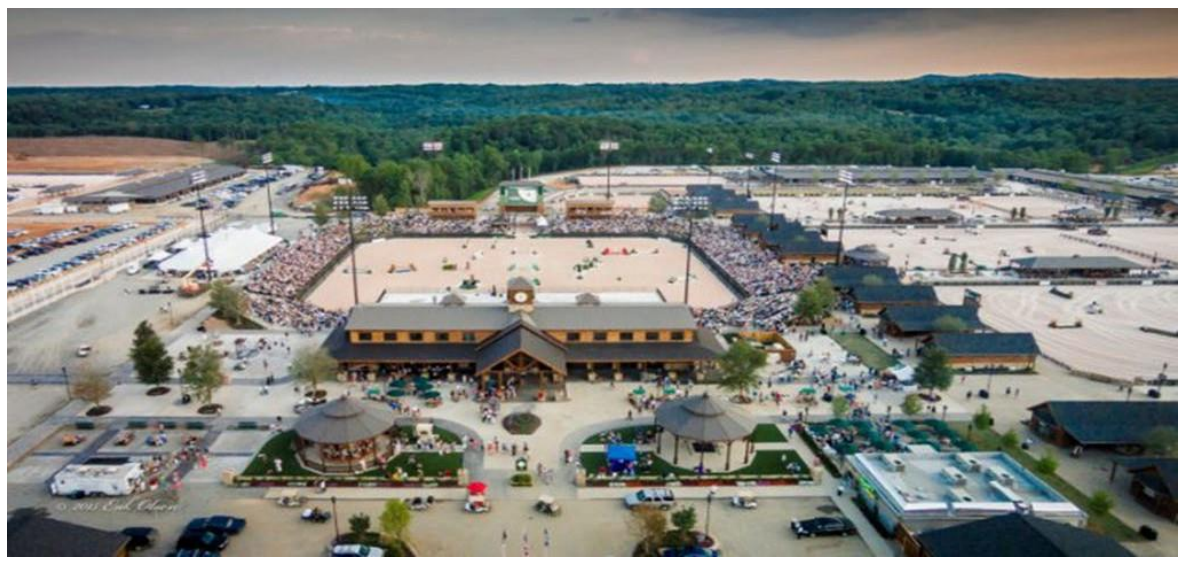

Figure 5 